BULLETIN OF THE

AMERICAN MATHEMATICAL SOCIETY

Volume 77, Number 3, May 1971

\title{
A RELATION BETWEEN TWO SIMPLICIAL ALGEBRAIC $K$-THEORIES
}

\author{
BY S. M. GERSTEN AND D. L. RECTOR
}

Communicated by Raoul Bott, November 2, 1970

There is a proliferation of proposed algebraic $K$-theories [5], [6], [8], [11], [12], [13], [15] and one of the present authors can share the blame for three of them. However some rather striking relationships have been found which indicate that the various $K$-theories, while not the same, are at any rate comparable. This note describes a relation between the $K$-theory proposed by Quillen [13], which has the advantage of computability using powerful techniques of the homology of groups, and that $K$-theory defined axiomatically in [8] and constructed semisimplicially in [5], which possesses extremely pleasant functorial properties. It is our hope that this connection will be useful in computing the $K$-theory of [8], and thus eventually the stable $K$-theory [7] which is analogous, in this rarefied setting of rings, with stable homotopy theory.

We begin by recalling (in slightly different form from [13]) Quillen's construction. For any ring $R$, one forms $\boldsymbol{Z}_{\infty} \bar{W}(\mathrm{Gl}(R))$. Here $\mathrm{Gl}(R)$ is regarded as a (constant) simplicial group, $\bar{W}$ is the simplicial classifying space, $[10$, p. 87$]$, and $\boldsymbol{Z}_{\infty}$ is the integral completion functor of Bousfield and $\operatorname{Kan}[2]$. Then $K_{i}^{Q}(R)=\pi_{i}\left(\boldsymbol{Z}_{\infty} \bar{W} \mathrm{Gl}(R)\right), i \geqq 1$, where the superscript refers to the author.

In order to give the simplicial definition of [5] of the $K$-theory of [8], we recall some terminology. One works in the category ring of rings (without unit) and one lets $E$ be the endomorphism of ring, $E R=t R[t]$, the path ring. The morphisms $\epsilon: E \rightarrow I, \mu \rightarrow E^{2}$ given by

$$
\begin{array}{rlrl}
\epsilon_{R}: E R & =t R[t] \rightarrow R, & \text { "t } & \rightarrow 1, " \text { and } \\
\mu_{R}: E R & =t R[t] \rightarrow t u R[t, u]=E^{2} R, \quad t \rightarrow t u,
\end{array}
$$

give rise to the cotriple $(E, \epsilon, \mu)$ in ring. Let $\bar{E} R$ be the augmented semisimplicial complex, $(\bar{E} R)_{n}=E^{n+2} R, n \geqq-1$, constructed from this cotriple, and set

$$
K^{-i}(R)=\tilde{\pi}_{i-2}(\mathrm{Gl}(\bar{E} R)), \quad i \geqq 1 .
$$

The upper indexing is motivated by topological considerations, and

AMS 1970 subject classifications. Primary 18F25, 55B15, 16A54, 13D15, 55F50.

$K e y$ words and phrases. Algebraic $K$-theory, the functor $Z_{\infty}$, nilpotent spaces, path ring, simplicial group, cotriple. 
$\tilde{\pi}$ refers to the "augmented" homotopy groups [5] $\left(\tilde{\pi}_{i}=\pi_{i}\right.$ for $i \geqq 1$, the augmentation entering for $i<1$ because of the extra face operator $\left.\epsilon:(\bar{E} R)_{0} \rightarrow(\bar{E} R)_{-1}\right)$.

Consider now the cotriple $(P, \epsilon, \mu)$ in ring where $P R=R[t], \epsilon_{R}: P R$ $\rightarrow R$ is given by " $t \rightarrow 1$ " and $\mu_{R}: P R=R[t] \rightarrow P^{2} R=R[t, u]$ is given by $t \rightarrow t u$. Let $\bar{P} R$ be the associated augmented semisimplicial complex. Then there is a canonical map

$$
\text { ı: } \mathrm{Gl}(\bar{E} R) \rightarrow \mathrm{Gl}(\bar{P} R) \text {. }
$$

Also, the complex $\operatorname{Gl}(\bar{P} R)$ is acyclic, so if $H$ is the homogeneous space of the inclusion $\iota$, then $H \simeq \bar{W} \mathrm{Gl}(\bar{E} R)$. We can however identify $H$ explicitly.

Note that $(\bar{P} R)_{n}=P^{n+1} R=R\left[t_{0}, \cdots, t_{n}\right]$ and $(\bar{E} R)_{n}=\left(t_{0} \cdots t_{n}\right)$ $\cdot R\left[t_{0}, \cdots, t_{n}\right]$. Thus we have a short exact sequence of rings

$$
(\bar{E} R)_{n} \rightarrow(\bar{P} R)_{n} \rightarrow Q(R)_{n},
$$

where $Q(R)_{n}=R\left[t_{0}, \cdots, t_{n}\right] /\left(t_{0} \cdots t_{n}\right), n \geqq 0 . Q(R)$ is a simplicial ring, and since $\mathrm{Gl}$ is left exact, we have an exact sequence of simplicial groups

$$
1 \rightarrow \mathrm{Gl}(\bar{E} R) \stackrel{\iota}{\rightarrow} \mathrm{Gl}(\bar{P} R) \stackrel{j}{\rightarrow} Q(R) .
$$

TheOREM 1. The canonical map $H \rightarrow Q R$ is an isomorphism of simplicial groups. In particular, the map $j$ above is surjective.

Note that $Q(R)_{0}=\operatorname{Gl}\left(R\left[t_{0}\right] /\left(t_{0}\right)\right)=\operatorname{Gl}(R)$. Thus we have an imbedding of the constant complex $\operatorname{Gl}(R) \stackrel{\leftrightarrow}{\rightarrow} Q(R)$ and hence a map

$$
\boldsymbol{Z}_{\infty} \bar{W}(\alpha): \boldsymbol{Z}_{\infty} \bar{W} \mathrm{Gl}(R) \rightarrow \boldsymbol{Z}_{\infty} \bar{W} Q(R) .
$$

Note that by Theorem $1, \operatorname{Gl}(\bar{E} R)$ is the "second loop group" of $\bar{W} Q(R)$, so we can identify $\pi_{i} \bar{W} Q(R)=K^{-i}(R), i \geqq 1$. In order to proceed further we need

THEOREM 2. The action of $\pi_{i} \bar{W} Q(R)$ on $\pi_{n} \bar{W} Q(R)$ is trivial. In particular, $\bar{W} Q(R)$ is "nilpotent" in the terminology of Bousfield and Kan.

This is proved by translating the problem to showing that the action of $\mathrm{Gl}(R)$ on the augmented homotopy groups of $\operatorname{Gl}(\bar{E} R)$ is trivial. This in turn is a generalization of the classical Whitehead lemma, which implies the statement of Theorem 2 for $\tilde{\pi}_{-1}$.

Corollary. [2]. For all $i$ we have

$$
K^{-i}(R) \cong \pi_{i}(\bar{W} Q R) \cong \pi_{i}\left(Z_{\infty} \bar{W} Q R\right),
$$


where the last isomorphism is induced by the canonical map $\bar{W} Q R$ $\rightarrow \boldsymbol{Z}_{\infty} \bar{W} Q R$.

\section{CoROLlary. The map}

$$
\boldsymbol{Z}_{\infty} \bar{W}(\alpha): \boldsymbol{Z}_{\infty} \bar{W} \mathrm{Gl}(R) \rightarrow \boldsymbol{Z}_{\infty} \bar{W} Q R
$$

induces natural homomorphisms $\alpha_{i}: K_{i}^{Q}(R) \rightarrow K^{-i}(R)$ for all $i \geqq 1$.

In low dimensions it is possible to identify $\alpha_{i}$. Namely, $\alpha_{1}$ is always surjective and corresponds to "reduction modulo unipotents." If $R$ is (left) regular, then $\alpha_{1}$ is an isomorphism by a result of Bass, Heller, and Swan [1] and $\alpha_{2}$ is surjective by [5, Theorem 6.1]. If $k$ is a finite field and $R=k(t)$, then $\alpha_{2}$ is known to be an isomorphism [4]. Also, if $R$ is the rationals, one knows that $\alpha_{2}$ is an isomorphism [9].

\section{REFERENCES}

1. H. Bass, A. Heller and R. G. Swan, The Whitehead group of a polynomial extension, Inst. Hautes Études Sci. Publ. Math. No. 22 (1964), 61-79. MR 30 \#4806.

2. A. K. Bousfield and D. M. Kan, Homotopy with respect to a ring, Proc. Sympos. Pure Math., vol. 22, Amer. Math. Soc., Providence, R. I. (to appear).

3. - The homotopy spectral sequence of a space with coefficients in a ring (preprint).

4. S. M. Gersten, $K$-theoretic interpretation of tame symbols on $k(t)$, Bull. Amer. Math. Soc. 76 (1970), 1073-1076.

5. - On Mayer-Vietoris functors and algebraic K-theory, J. Algebra (to appear).

6. - On the functor $K_{2}$. I, J. Algebra (to appear).

7. - Stable $K$-theory of discrete rings (to appear).

8. M. Karoubi and O. Villamayor, Foncteurs $K^{n}$ en algèbre et en topologie, C. R. Acad. Sci. Paris Sér. A-B 269 (1969), A416-A419. MR 40 \#4944.

9. - Groupes d'homotopie algébriques et foncteurs $K^{-n}$, Institute de Recherche Mathématique Avancée, CNRS, Strasbourg (preprint).

10. J. P. May, Simplicial objects in algebraic topology, Van Nostrand Math. Studies, no. 11, Van Nostrand, Princeton, N. J., 1967. MR 36 \#5942.

11. J. Milnor, Algebraic K-theory and quadratic forms, Invent. Math. 9 (1970), 318-344.

12. A. Nobile and O. Villamayor, Sur la K-thêorie algébrique, Ann. Sci. École Norm. Sup. (4) 1 (1968), 581-616. MR 39 \#1526.

13. D. Quillen, Cohomology of groups (preprint).

14. - The K-theory associated to a finite field. I (preprint).

15. R. G. Swan, Non-abelian homological algebra and K-theory, Proc. Sympos. Pure Math., vol. 17, Amer. Math. Soc., Providence, R. I., 1970, pp. 88-123.

Rice University, Houston, Texas 77001 